\title{
Stability of Gauss-Bonnet black holes in Anti-de-Sitter space-time against scalar field condensation
}

\author{
Yves Brihaye $^{* \ddagger}$ and Betti Hartmann ${ }^{\dagger}$ \\ ${ }^{\ddagger}$ Physique-Mathématique, Universite de Mons-Hainaut, 7000 Mons, Belgium \\ ${ }^{\dagger}$ School of Engineering and Science, Jacobs University Bremen, 28759 Bremen, Germany
}

\begin{abstract}
We study the stability of static, hyperbolic Gauss-Bonnet black holes in $(4+1)$-dimensional Anti-deSitter (AdS) space-time against the formation of scalar hair. Close to extremality the black holes possess a near-horizon topology of $\mathrm{AdS}_{2} \times H^{3}$ such that within a certain range of the scalar field mass one would expect that they become unstable to the condensation of an uncharged scalar field. We confirm this numerically and observe that there exists a family of hairy black hole solutions labelled by the number of nodes of the scalar field function. We construct explicit examples of solutions with a scalar field that possesses zero nodes, one node and two nodes, respectively, and show that the solutions with nodes persist in the limit of Einstein gravity, i.e. for vanishing Gauss-Bonnet coupling. We observe that the interval of the mass for which scalar field condensation appears decreases with increasing Gauss-Bonnet coupling and/or with increasing node number.
\end{abstract}

PACS Numbers: 04.70.-s, 04.50.Gh, 11.25.Tq

\section{Introduction}

Higher curvature corrections appear naturally in the low energy effective action of string theory [1]. In more than four dimensions the quadratic correction is often chosen to be the Gauss-Bonnet (GB) term, which has the property that the equations of motion are still second order in derivatives of the metric functions. As such explicit solutions of the equations of motion are known. The first example of static, spherically symmetric and asymptotically flat black hole solutions in GB gravity were given for the uncharged case in 2, 3, and for the charged case in [4]. Moreover, the corresponding solutions in asymptotically Anti-de Sitter (AdS) [5, 6, 7, 8] as well as de Sitter (dS) space-times 9 have been studied. In most cases, black holes not only with spherical $(k=1)$, but also with flat $(k=0)$ and hyperbolic $(k=-1)$ horizon topology have been considered. Moreover, the thermodynamics of these black holes has been studied in detail [7, 8, 10] and the question of negative entropy for certain GB black holes in dS and AdS has been discussed [6, 11.

The gravity-gauge theory duality [12] has attracted a lot of attention in the past years. The most famous example is the AdS/CFT correspondence [13] which states that a gravity theory in a $d$-dimensional Anti-de Sitter (AdS) space-time is equivalent to a Conformal Field Theory (CFT) on the $(d-1)$-dimensional boundary of AdS. Recently, this correspondence has been used to describe so-called holographic superconductors with the help of black holes in higher dimensional AdS space-time [14, 15, 16, 17. In most cases (3+1)-dimensional black holes with planar horizons $(k=0)$ were chosen to account for the fact that high temperature superconductivity is mainly associated to 2-dimensional layers within the material. The basic idea is that at low temperatures

\footnotetext{
*email: yves.brihaye@umons.ac.be

†email: b.hartmann@jacobs-university.de
} 
a planar black hole in asymptotically AdS becomes unstable to the condensation of a charged scalar field. The hairy black hole is the gravity dual of the superconductor. The main point here is that this instability occurs due to the fact that the scalar field is charged and its effective mass drops below the BreitenlohnerFreedman (BF) bound [18] for sufficiently low temperature of the black hole hence spontaneously breaking the U(1) gauge symmetry. Surprisingly, however, the scalar condensation can also occur for uncharged scalar fields in the $d$-dimensional planar Reissner-Nordström-AdS (RNAdS) black hole space-time [15. This is a new type of instability that is not connected to a spontaneous symmetry breaking as in the charged case. Rather it is related to the fact that the planar RNAdS black hole possesses an extremal limit with vanishing Hawking temperature and near-horizon geometry $\mathrm{AdS}_{2} \times \mathbb{R}^{d-2}$ (with $d \geq 4$ ) [19, 20, 21. For scalar field masses larger than the $d$-dimensional $\mathrm{BF}$ bound, but smaller than the 2-dimensional $\mathrm{BF}$ bound the near-horizon geometry becomes unstable to the formation of scalar hair, while the asymptotic $\mathrm{AdS}_{d}$ remains stable [15. The fact that the near-horizon geometry of extremal black holes is a topological product of two manifolds with constant curvature has let to the development of the entropy function formalism [22, 23, 24]. In [25] the question of the condensation of a uncharged scalar field on uncharged black holes in $(4+1)$ dimensions has been addressed. As a toy model for the rotating case, static black holes with hyperbolic horizons $(k=-1)$ were discussed. In contrast to the uncharged, static black holes with flat $(k=0)$ or spherical $(k=1)$ horizon topology hyperbolic black holes possess an extremal limit with near-horizon geometry $\mathrm{AdS}_{2} \times H^{3}$ and it has been shown by numerical construction that the black holes form scalar hair close to extremality.

Interestingly, there seems to be a contradiction between the holographic superconductor approach and the Coleman-Mermin-Wagner theorem [26] which forbids spontaneous symmetry breaking in $(2+1)$ dimensions at finite temperature. Consequently, it has been suggested that higher curvature corrections and in particular GB terms should be included on the gravity side and holographic GB superconductors in $(3+1)$ dimensions have been studied [27. However, though the critical temperature gets lowered when including GB terms, condensation cannot be suppressed - not even when including backreaction [28, 29].

In this paper, we are interested in the condensation of an uncharged scalar field on an uncharged hyperbolic GB black hole in (4+1)-dimensional AdS. These black holes have been shown to possess a near-horizon geometry of $\mathrm{AdS}_{2} \times H^{3}$ in the extremal limit [30. In accordance with the results in the Einstein gravity limit [25] we would thus expect that the hyperbolic GBAdS black holes would become unstable for scalar field masses below the 2-dimensional BF bound. We construct explicit examples of hairy GBAdS black holes and study their properties. We also reinvestigate the Einstein gravity limited studied before and show that next to the solutions found in 25. their exists a family of hairy black hole solutions labelled by the number of nodes of the scalar field function. Our paper is organised as follows: in Section 2 we give our model, the equations of motions, the boundary conditions and discuss the black hole properties as well as exact solutions of the equations of motion. In Section 3 we give our numerical results, first for the linear case and then for the non-linear case. We conclude in Section 4.

\section{The Model}

In this paper, we study Gauss-Bonnet (GB) black holes in $(4+1)$-dimensional Anti-de Sitter (AdS) space-time against condensation of an uncharged scalar field. The action that we consider reads :

$$
S=\frac{1}{16 \pi G} \int d^{5} x \sqrt{-g}\left(R-2 \Lambda+\frac{\alpha}{2}\left(R^{M N K L} R_{M N K L}-4 R^{M N} R_{M N}+R^{2}\right)+16 \pi G \mathcal{L}_{\text {matter }}\right),
$$

where $\Lambda=-6 / L^{2}$ is the cosmological constant, $L$ denotes the AdS radius and $G$ is Newton's constant. $\alpha$ is the Gauss-Bonnet coupling that has dimension (length) ${ }^{2}$ and is bounded from above $\alpha \leq L^{2} / 4$, where $\alpha=L^{2} / 4$ is the Chern-Simons limit. $R, R_{M N}$ and $R_{M N K L}, M, N, K, L=0,1,2,3,4$ denote the Ricci scalar, the Ricci tensor and the Riemann tensor, respectively, while $g$ is the determinant of the metric. The matter Lagrangian $\mathcal{L}_{\text {matter }}$ of a real valued scalar field with mass $m^{2}$ reads :

$$
\mathcal{L}_{\text {matter }}=-\left(\partial_{M} \psi\right) \partial^{M} \psi-m^{2} \psi^{2} \quad, \quad M=0,1,2,3,4 .
$$


In the following, we want to study static, hyperbolic black holes. The Ansatz for the metric hence reads :

$$
d s^{2}=-f(r) a^{2}(r) d t^{2}+\frac{1}{f(r)} d r^{2}+\frac{r^{2}}{L^{2}} d \Xi_{3}^{2}
$$

where $d \Xi_{3}^{2}$ is the line element of a 3-dimensional hyperbolic space with curvature $k=-1$ and Ricci scalar $R=-6$. The coupled Einstein and Euler-Lagrange equations are obtained from the variation of the action with respect to the matter and metric fields, respectively. They read :

$$
\begin{aligned}
f^{\prime} & =\frac{2 r\left(-1-f+\frac{2 r^{2}}{L^{2}}\right)}{r^{2}-2 \alpha(1+f)}-\gamma r^{3}\left(\frac{m^{2} \psi^{2}+f \psi^{\prime 2}}{r^{2}-2 \alpha(1+f)}\right), \\
a^{\prime} & =\gamma \frac{r^{3} a \psi^{\prime 2}}{r^{2}-2 \alpha(1+f)}, \\
\psi^{\prime \prime} & =-\left(\frac{3}{r}+\frac{f^{\prime}}{f}+\frac{a^{\prime}}{a}\right) \psi^{\prime}+\frac{m^{2}}{f} \psi,
\end{aligned}
$$

where we have used the abbreviation $\gamma=\frac{16}{3} \pi G$. Here and in the following the prime denotes the derivative with respect to $r$. Note that the equations (4)-(6) can be rescaled as follows $\psi \rightarrow \psi / \sqrt{\gamma}$ for $\gamma \neq 0$. We can hence set $\gamma=1$ without loss of generality. Different choices of $\gamma$ will simply lead to a different choice of normalisation of $\psi$. For the linear case $\gamma=0$ the normalisation has to be fixed by hand.

The set of coupled, non-linear ordinary differential equations (4)-(6) has to be solved numerically subject to appropriate boundary conditions. At the event horizon $r=r_{+}$these read

$$
f\left(r_{+}\right)=0
$$

with $a\left(r_{+}\right)$finite. Moreover, in order for the scalar field to be regular at the horizon we need to impose

$$
\psi^{\prime}\left(r_{+}\right)=\left.\frac{m^{2} \psi\left(r^{2}-2 \alpha\right)}{-2 r+4 r^{3} / L^{2}-\gamma r^{3} m^{2} \psi^{2}}\right|_{r=r_{+}} .
$$

Asymptotically, we want the scalar field to have the following behaviour

$$
\psi(r \gg 1)=\frac{\psi_{+}}{r^{\Delta_{+}}}+\frac{\psi_{-}}{r^{\Delta_{-}}}
$$

with

$$
\Delta_{ \pm}=2 \pm \sqrt{4+m^{2} L_{\text {eff }}^{2}} \quad, \quad L_{\text {eff }}^{2} \equiv \frac{2 \alpha}{1-\sqrt{1-4 \alpha / L^{2}}} .
$$

$L_{\text {eff }}$ is the effective AdS radius, which behaves like $L_{\text {eff }}^{2} \sim L^{2}\left(1-\alpha / L^{2}+O\left(\alpha^{2}\right)\right)$ for small $\alpha$ and becomes equal to $L_{\text {eff }}^{2}=L^{2} / 2$ in the Chern-Simons limit $\alpha=L^{2} / 4$. Hence, the effective AdS radius decreases with increasing $\alpha$. Finally, the behaviour of the metric functions at $r \gg 1$ is given by

$$
f(r \gg 1)=-1+\frac{r^{2}}{L_{\mathrm{eff}}^{2}}+\frac{f_{2}}{r^{2}}+O\left(r^{-4}\right), a(r \gg 1)=1+\frac{a_{4}}{r^{4}}+O\left(r^{-6}\right),
$$

where $f_{2}$ and $a_{4}$ are constants that have to be determined numerically.

\subsection{Black hole properties}

The Hawking temperature $T_{\mathrm{H}}$ and entropy $S$ of the black holes studied in this paper read 30.

$$
T_{\mathrm{H}}=\frac{f^{\prime}\left(r_{+}\right) a\left(r_{+}\right)}{4 \pi} \quad, \quad \frac{S}{V_{3}}=\frac{r_{+}^{3}}{4 G}\left(1-\frac{6 \alpha}{r_{+}^{2}}\right),
$$


where $V_{3}$ corresponds to the volume of the 3 -dimensional hyperbolic space with line element $d \Xi_{3}^{2}$, which we assume to be compactified. In the following we will set $V_{3}=1$ hence assuming that all quantities containing this factor are given per unit hyperboloid volume. The specific heat is given by $C=T_{\mathrm{H}}\left(\partial S / \partial T_{\mathrm{H}}\right)$. For $C>(<) 0$ the black holes are thermodynamically stable (unstable).

We will also be interested in the energy $E$ of the solutions. Using the counterterm approach by Brown and York [31, 32] we find that the energy $E$ can be expressed in terms of the expansion coefficients of the metric functions $f(r)$ and $a(r)$ (see (11)) as follows

$$
\frac{16 \pi G E}{V_{3}}=\sqrt{1-\frac{\alpha}{L^{2}}}\left(-3 f_{2}-8 \frac{a_{4}}{L_{\text {eff }}^{2}}\right) .
$$

The main feature that distinguishes Gauss-Bonnet-Anti-de Sitter (GBAdS) and Schwarzschild-Anti-de Sitter (SAdS) black holes with hyperbolic horizons from those with flat or spherical horizons is that an extremal limit with $T_{\mathrm{H}}=0$ exists (see also 2.2). Close to extremality the horizon topology is $\operatorname{AdS}_{2} \times H^{3}$ with radius of the $\mathrm{AdS}_{2}$ given by $R$ [30. Now the Breitenlohner-Freedman bound 18 for a scalar field in $d$-dimensional AdS is

$$
m^{2} \geq m_{\mathrm{BF}, \mathrm{d}}^{2}=-\frac{(d-1)^{2}}{4 L_{\mathrm{eff}}^{2}} .
$$

Using the entropy function formalism 22, 23, 24, we find that the radius $R$ of the $\mathrm{AdS}_{2}$ in this case reads (see Appendix for details on the calculation for horizons with spherical, hyperbolic and flat horizon topology)

$$
R=\sqrt{\frac{L^{2}}{4}-\alpha} .
$$

It is obvious from this formula that we need to require $L^{2}>4 \alpha$ in order to find an extremal solution whose near-horizon topology possesses an $\mathrm{AdS}_{2}$-factor. In the Chern-Simons limit $L^{2}=4 \alpha$, the radius of the $\mathrm{AdS}_{2}$ simply vanishes. Hence for scalar masses satisfying

$$
-\frac{4}{L_{\mathrm{eff}}^{2}} \leq m^{2} \leq-\frac{1}{4 R^{2}}=-\frac{1}{L^{2}-4 \alpha}
$$

we find that the asymptotic $\mathrm{AdS}_{5}$ is stable, while the near horizon $\mathrm{AdS}_{2}$ is unstable. We would thus expect that the GB black hole becomes unstable to the formation of scalar hair in this range of the parameter $m$.

\subsection{Exact solutions}

In the case $\gamma=0$, i.e. when the scalar and gravity equations are decoupled, there exists an explicit solution to the equations [2, 3, 5]. The metric functions of this Gauss-Bonnet-Anti-de Sitter (GBAdS) solutions read

$$
f(r)=-1+\frac{r^{2}}{2 \alpha}\left(1-\sqrt{1-\frac{4 \alpha}{L^{2}}+\frac{4 \alpha M}{r^{4}}}\right)=-1+\frac{r^{2}}{2 \alpha}\left[1-\sqrt{1+\frac{4 \alpha}{L^{2}}\left(\frac{r_{+}^{4}}{r^{4}}-1\right)+\frac{4 \alpha}{r^{4}}\left(\alpha-r_{+}^{2}\right)}\right]
$$

and $a(r) \equiv 1 . M$ is an integration constant that is connected to the Arnowitt-Deser-Misner (ADM) mass of the solution $M_{\mathrm{ADM}}$ by $M_{\mathrm{ADM}}=3 M V_{3} /(16 \pi G)$ [10]. In the last equality in (17) we have used the relation between the event horizon $r_{+}$and $M$, where the horizons of the space-time are given by

$$
r_{ \pm}=\frac{1}{2} \sqrt{2 L^{2} \pm 2 \sqrt{L^{4}-4 \alpha L^{2}+4 M L^{2}}}
$$

such that

$$
M=\alpha+\frac{r_{+}^{4}}{L^{2}}-r_{+}^{2}
$$


In the $\gamma=0$ limit, the scalar field equation (6) becomes linear and describes a scalar field in the background space-time with metric functions given by (17) and $a(r) \equiv 1$. Note that $\psi(r) \equiv 0$ together with (17) is a solution to the full set of equations (4)-(6) for generic $\gamma$. The Hawking temperature of this black hole reads

$$
T_{\mathrm{H}}=\frac{1}{4 \pi} f^{\prime}\left(r_{+}\right) a\left(r_{+}\right)=\frac{-r_{+}+\frac{2 r_{+}^{3}}{L^{2}}}{2 \pi\left(r_{+}^{2}-2 \alpha\right)} .
$$

The extremal solution with $T_{\mathrm{H}}=0$ has horizon $r_{+}^{(\mathrm{ex})}=L / \sqrt{2}$ and mass parameter $M^{(\mathrm{ex})}=\alpha-L^{2} / 4$, which is always negative for $\alpha<L^{2} / 4$. In order for the temperature $T_{\mathrm{H}}$ to be non-negative, we have to require that $r_{+} \geq r_{+}^{(\text {ex })} \geq \sqrt{2 \alpha}$ which is always fulfilled for $\alpha<L^{2} / 4$. The energy of the GBAdS black hole solutions is given by

$$
E=\sqrt{1-\frac{\alpha}{L^{2}}} M_{\mathrm{ADM}}
$$

There are solutions with $E=M=M_{\mathrm{ADM}}=0$. These are vacuum solutions, which have horizons at $r_{+}=r_{+, 0}$ with

$$
\frac{r_{+, 0}^{2}}{L^{2}}=\frac{1}{2}\left(1+\sqrt{1-\frac{\alpha}{4 L^{2}}}\right)
$$

such that $r_{+, 0}>r_{+}^{(\mathrm{ex})}$. Black holes with $r_{+}<r_{+, 0}$ have negative energy and the extremal solution has the lowest energy. The $M=0$ solution is special as it is locally isometric to $\mathrm{AdS}_{5}$ with effective $\operatorname{AdS}$ radius $L_{\text {eff }}$. When the $\mathrm{AdS}_{5} \mathrm{BF}$ bound is satisfied (see (14)), i.e. when $\Delta \equiv \Delta_{ \pm}=2$ there is an exact solution to the equations of motion with $\psi(r)=L_{\text {eff }}^{2} / r^{2}$. Very similar to the $\alpha=0$ case [25] this does, however, not signal an instability of $\mathrm{AdS}_{5}$ since our solution covers only part of $\mathrm{AdS}_{5}$.

The entropy is given by the expression in (12). Note that this explicit form can be derived from the first law of black hole thermodynamics $d M_{\mathrm{ADM}}=T d S$ by using (19). Since the entropy can become negative it has been suggested in [11] that the entropy $S$ of the vacuum solution with $r_{+}=r_{+, 0}$ should be substracted from this expression assigning entropy $S=0$ to the vacuum solution such that

$$
S=\int_{r_{+}, 0}^{r_{+}} T^{-1}\left(\frac{\partial M_{\mathrm{ADM}}}{\partial r_{+}}\right) d r_{+} .
$$

The specific heat $C$ can be calculated explicitly and reads

$$
4 G V_{3} C=\frac{3 r_{+}\left(2 \alpha-r_{+}^{2}\right)^{2}\left(2 r_{+}^{2}-L^{2}\right)}{2 r_{+}^{4}+r_{+}^{2}\left(L^{2}-12 \alpha\right)+2 \alpha L^{2}} .
$$

This is always positive for $r_{+} \geq r_{+}^{(\mathrm{ex})}$ and $\alpha<L^{2} / 4$. Hence, the black holes are thermodynamically stable.

There are two important limits of (17). The first is the Einstein gravity limit $\alpha=0$, whose stability with respect to the formation of scalar hair has been discussed in detail in 25. In this case the equations admit Schwarzschild-Anti-de Sitter (SAdS) solutions with metric function $f(r)$

$$
f(r)=-1+\frac{r^{2}}{L^{2}}-\frac{M}{r^{2}}=-1+\frac{r^{2}}{L^{2}}+\frac{r_{+}^{2}}{r^{2}}\left(1-\frac{r_{+}^{2}}{L^{2}}\right) .
$$

This solution has an extremal limit with $r_{+}=r_{+}^{(\mathrm{ex})}=L / \sqrt{2}$ and mass parameter $M^{(\mathrm{ex})}=-L^{2} / 4$. The fact that the near-horizon topology of the SAdS solution is $\mathrm{AdS}_{2} \times H^{3}$ was used in 25 to show that these black holes become unstable to the formation of scalar hair on the horizon for specific values of the mass parameter $m$.

The other limit is the Chern-Simons limit $\alpha=L^{2} / 4$ with

$$
f(r)=\frac{2}{L^{2}}\left(r^{2}-r_{+}^{2}\right) .
$$

The solution with $T_{\mathrm{H}}=0$ and $r_{+}^{(\mathrm{ex})}=L / \sqrt{2}$ is special in this case [10] since $M^{(\mathrm{ex})}=0$. Along with [10] we will hence restrict ourselves to $\alpha<L^{2} / 4$ in the following, also because - as mentioned above - the $\mathrm{AdS}_{2}$ in the Chern-Simons limit has vanishing AdS radius $R$. 


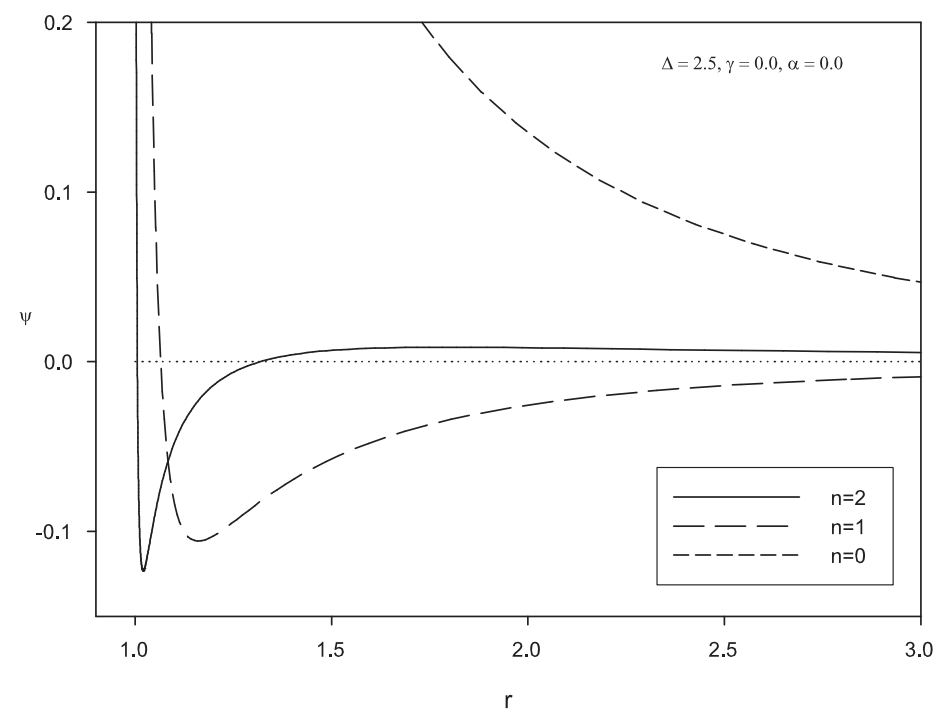

Figure 1: The profile of the scalar field function $\psi(r)$ is shown for $\Delta=2.5, \gamma=0$ and $\alpha=0$. Next to the fundamental solution without nodes $(n=0)$ there exist solutions with nodes. We here present the solution with $n=1$ nodes and that with $n=2$ nodes, respectively.

\section{Numerical results}

We have solved the set of equations (4)-(6) subject to the appropriate boundary conditions numerically using a collocation method for ordinary differential equations 33 . The relative errors of the solutions are on the order of $10^{-10}-10^{-6}$. In the following we will fix $r_{+}=1$ without loss of generality and restrict ourselves to $\alpha<0.5$ to ensure that the Hawking temperature $T_{\mathrm{H}}$ in the linear case $\gamma=0$ is positive (see (20)). We will also limit our analysis to $\Delta \geq 1$ to ensure that the modes are normalisable.

Along with [25] we choose the scalar field to fall off like $\psi(r \rightarrow \infty)=\psi_{0} r^{-\Delta}$. For $\Delta \equiv \Delta_{+} \geq 2$ we have $\psi_{-}=0, \psi_{+} \equiv \psi_{0} \neq 0$, while for $\Delta \equiv \Delta_{-}<2$ we let $\psi_{+}=0, \psi_{-} \equiv \psi_{0} \neq 0$. The case $\Delta \equiv \Delta_{ \pm}$corresponds to the saturation of the 5 -dimensional $\mathrm{BF}$ bound. Next to these conditions we have to impose the regularity condition (8). Since the equations are invariant under the rescaling $\psi(r) \rightarrow \psi(r) / \sqrt{\gamma}$ the normalisation of $\psi(r)$ has to be fixed in addition. In the following we will choose $\psi\left(r_{+}\right)=1$. These are a total of three boundary conditions to be fulfilled for the second order differential equation (6). Regular solutions corresponding to a fixed value of $\Delta$ will hence only exist for specific values of $r_{+} / L_{\text {eff }} \equiv 1 / L_{\text {eff }}$. In the following, we will let our numerical routine determine $L_{\text {eff }}^{2}$ for a fixed value of $\Delta$ subject to the three boundary conditions mentioned above. Clearly, the parameters $L^{2}$ and $m^{2}$ can then be reconstructed from the numerical value of $L_{\text {eff }}^{2}$.

\subsection{Linear case: $\gamma=0$}

We have first studied the case $\gamma=0$, in which (6) becomes linear and describes a scalar field in the background of the black hole solution given by (17) with $a(r) \equiv 1$.

To test our numerical technique we have reexamined the case $\alpha=0$ and recovered the results given in [25]. In addition to the hairy black hole solution constructed in [25], where the scalar field $\psi(r)$ is monotonically decreasing from $r=r_{+}$to $r=\infty$, we have been able to construct solutions where the scalar field possesses a number $n$ of nodes. This is shown in Fig 1 where we present $\psi(r)$ for $\Delta=2.5, n=0,1$, , respectively. We believe that solutions with an arbitrary number of nodes $n$ exist, but we did not attempt to construct the solutions for $n>2$. 


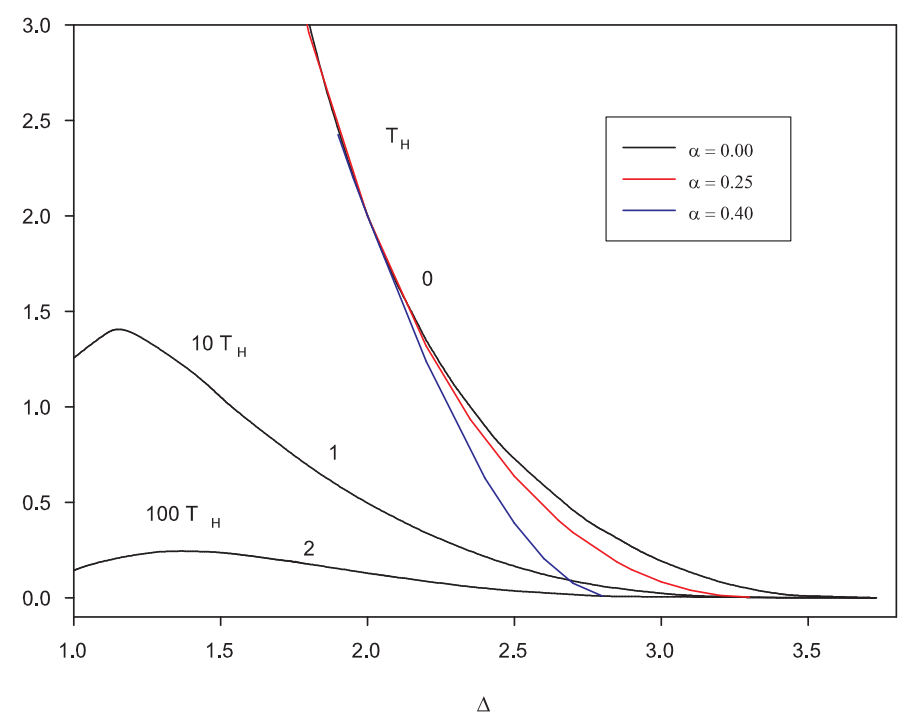

Figure 2: The Hawking temperature $T_{\mathrm{H}}$ as function of $\Delta$ for the $n=0,1,2$ SAdS solutions with $\alpha=0$ (black). The number of nodes $n$ is indexed by the numbers $0,1,2$, respectively, on the curves. We also give the temperature of the $n=0$ GBAdS black holes for $\alpha=0.25$ (red) and $\alpha=0.4$ (blue). Here $\gamma=0$.

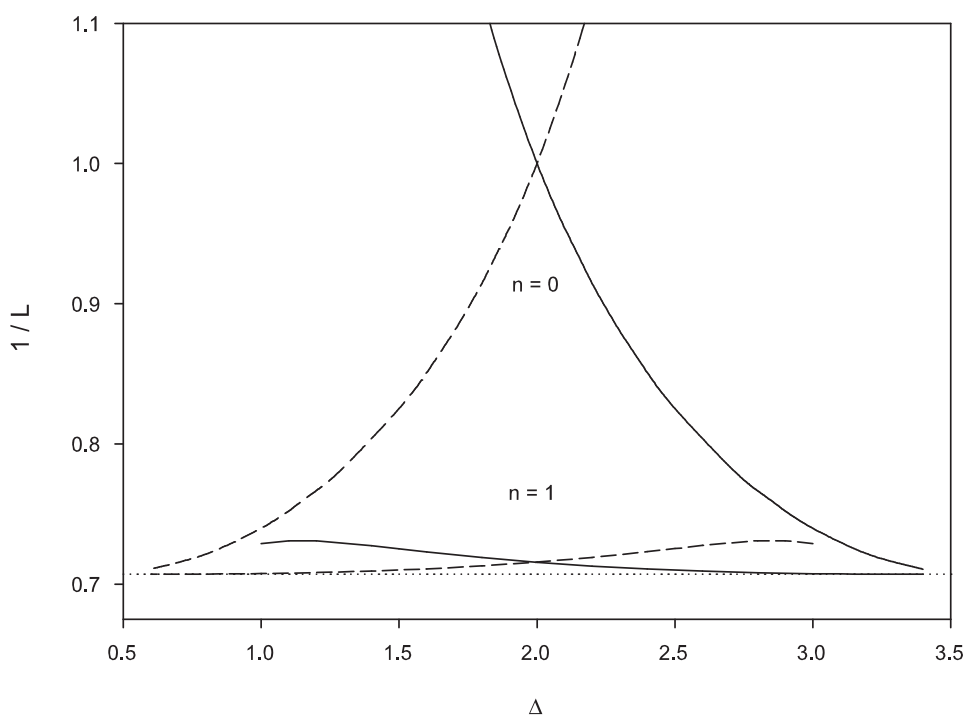

Figure 3: The value of $\frac{r_{+}}{L} \equiv \frac{1}{L}$ as function of $\Delta$ for the $n=0,1$ SAdS black holes with $\gamma=0$. We show the value of $\Delta \equiv \Delta_{+}$given by the solid and dashed curves with $\Delta>2$ and the value of $\Delta \equiv \Delta_{-}$given by the solid and dashed curves with $\Delta<2$. Here $\gamma=0$. 


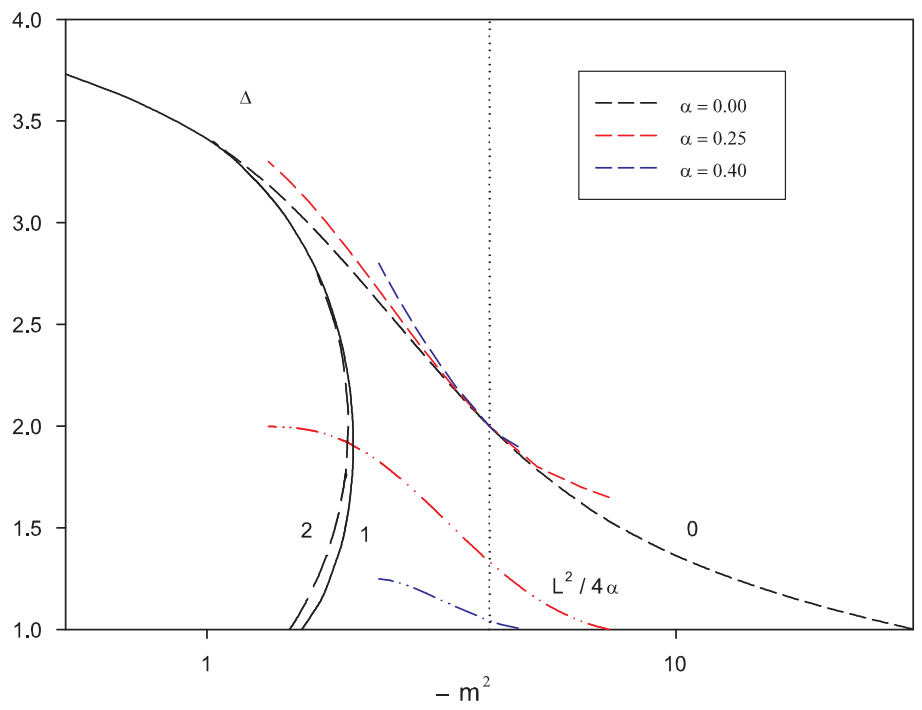

Figure 4: The value of $\Delta$ as function of the mass $-m^{2}$ for the $n=0,1,2$ (short-dashed, solid, long-dashed) SAdS black hole solutions (black). We also show the value of $\Delta$ for $n=0$ GBAdS black hole solutions for $\alpha=0.25$ (red short-dashed) and $\alpha=0.4$ (blue short-dashed), respectively. The value of $L^{2} / 4 \alpha$ is also given for $n=0$ GB black holes for $\alpha=0.25$ (red dotted-dashed) and $\alpha=0.4$ (blue dotted-dashed), respectively. The dotted vertical line corresponds to the saturation of the $\mathrm{AdS}_{5}$ BF bound with $m^{2}=-4 / L^{2} \equiv-4$ and $\Delta=2$ (see also Fig. 3). Here $\gamma=0$.

In Fig. 2 we give the temperature $T_{\mathrm{H}}$ of the hairy black holes for $n=0,1,2$ as function of $\Delta$. Our numerical results suggest that the solutions stop existing for $\Delta=2+\sqrt{3} \approx 3.732$. This agrees with the exact result when inserting the $\mathrm{AdS}_{2} \mathrm{BF}$ bound $m^{2}=-1 / L^{2}$ for $\alpha=0$ (see (16) ) into the expression for $\Delta$ (see (10)). This has already been observed in 25] for $n=0$, but we find that this remains valid for solutions with nodes. Fig. 2 clearly shows that the condensation of scalar fields with nodes happens at much lower temperatures than that of the scalar field without nodes. In fact, we find that the higher $n$ the lower the temperature has to be and hence the closer to the extremal solution we have to be to find the scalar field condensation instability.

In Fig 3 we give the value of $r_{+} / L \equiv 1 / L$ as function of $\Delta$ for the $n=0,1$ node solutions. The solid $n=0$ line was already presented in [25]. Note that for $\Delta>2$, this curve corresponds to $\Delta_{+}$, while for $\Delta<2$ this is $\Delta_{-}$. In addition to what was given in 25 we also give the results for the other normalisable mode which has $\Delta_{-}$for $\Delta<2$ and $\Delta_{+}$for $\Delta>2$, respectively. We observe that in analogy to what has been observed for the $n=0$ black holes [25] also $n=1$ black holes show an instability against scalar hair condensation only for $r_{+} / L \geq r_{+}^{(\mathrm{ex})} / L=\sqrt{2}$. This happens at the saturation of the $\mathrm{AdS}_{2} \mathrm{BF}$ bound with $\Delta \equiv \Delta_{+}=2+\sqrt{3}$ or $\Delta \equiv \Delta_{-}=2-\sqrt{3}$. Note, however, that this latter value would correspond to a non-normalisable mode since $\Delta<1$. The $\Delta_{+}$and $\Delta_{-}$curves meet at $\Delta=2$. This corresponds to the saturation of the 5 -dimensional $\mathrm{BF}$ bound with $m^{2}=-4 / L^{2}$ and for the $n=0$ solution happens at $r_{+}=L$, i.e. $M=0$. In $[25$ this was related to the existence of an exact solution to the equations of motion with $\psi(r)=L^{2} / r^{2}$ and $f(r)=-1+r^{2} / L^{2}$. This describes a scalar field in a space-time isometric to a part of $\mathrm{AdS}_{5}$. For the $n=1$ solutions we find that the $\Delta_{+}$and $\Delta_{-}$curves meet at $\Delta=2$, i.e. in the limit of saturation of the $\mathrm{AdS}_{5} \mathrm{BF}$ bound, but for $r_{+} / L \approx 0.715$. Hence the space-time is clearly not isometric to $\mathrm{AdS}_{5}$ in this case and we could not find any exact solution given in terms of powers of $r$.

Note that all black holes with $r_{+} / L<1$ have $M<0$. We observe, however, that in addition to what was discussed in 25] $n=0$ black holes with $r_{+} / L>1$ can also have a scalar field condensation instability with $\Delta>2$. These black holes have positive $M$ and hence positive energy $E$. We thus find that a scalar condensation 
instability with $\Delta>2$ does not only occur on SAdS black holes with negative energy $E$, but also on SAdS black holes with positive energy $E$. The $n=1$ black hole solutions exist only up to a maximal value of $r_{+} / L$. We find numerically that $r_{+} / L \lesssim 0.731$ and that the maximal value is attained at $\Delta=1.2$. Hence, scalar fields with nodes can only condensate on small black holes.

In Fig 4 we give $\Delta$ as function of $m^{2}$ for the $n=0,1,2$ solutions. We note that while for the $n=0$ solutions the mass range for which scalar condensation appears is quite large, this is different for the $n=1$ and $n=2$ node solutions. For all solutions, the maximal value of $m^{2}=m_{\max }^{2}$ corresponds to the saturation of the 2-dimensional BF bound $m^{2}=m_{\mathrm{BF}}^{2}=-1 / L^{2}$ at the extremal solution with $r_{+}=r_{+}^{(\mathrm{ex})}=L / \sqrt{2}$ (see discussion above). With our choice $r_{+}=1$ this corresponds to $m_{\max }^{2}=-0.5$. The minimal value of $m^{2}=m_{\min }^{2}$ on the other hand depends to the number of nodes $n$. With the restriction that $\Delta \geq 1$ we find $m_{\min }^{2} \approx-32.1$ with corresponding $r_{+} / L \equiv 1 / L \approx 3.20$ for $n=0, m_{\min }^{2} \approx-2.48$ with corresponding $r_{+} / L \equiv 1 / L \approx 0.72$ for $n=1$ and $m_{\min }^{2} \approx-2.01$ with corresponding $r_{+} / L \equiv 1 / L \approx 0.71$ for $n=2$. Note that the minimal value of $m^{2}$ is attained at $\Delta=1$ for the $n=0$ solutions, but at $\Delta \approx 2$ for the $n=1,2$ solutions. The value of $m^{2}$ at $\Delta=1$ is larger than $m_{\min }^{2}$ for the solutions with nodes and is $m_{\Delta=1}^{2} \approx-1.59$ for the $n=1$ solution and $m_{\Delta=1}^{2} \approx-1.50$ for the $n=2$ solution, respectively. We thus conclude that for a fixed value of the mass in the interval $m_{\min }^{2} \leq m^{2} \leq m_{\Delta=1}^{2}$ there exist two hairy black hole solutions which differ in their fall-off of the scalar field function on the AdS boundary. Moreover, the interval of the mass $m^{2}$ on which scalar condensation appears decreases with the increase of the number of nodes $n$.

Next, we have studied the influence of the Gauss-Bonnet term by letting $\alpha \neq 0$. We find that the solutions with nodes also exist in this case. However, the numerical values for the interesting quantities are very close to those for $\alpha=0$. This is why we do not present them here. We give the temperature $T_{\mathrm{H}}$ of the $n=0$ GBAdS solutions for $\alpha=0.25$ and $\alpha=0.4$ in Fig 2 . We observe that the solutions cease to exist for smaller values of $\Delta$ as compared to the $\alpha=0$ limit. This can be explained by considering (16) for $\alpha \neq 0$ and inserting $m^{2}=-1 /\left(L^{2}-4 \alpha\right)$ into the expression for $\Delta$. This is a complicated expression, but the effect can be seen easily when expanding $\Delta$ for small $\alpha$. This reads $\Delta \approx 2+\sqrt{3-3 \alpha / L^{2}-11 \alpha^{2} / L^{4}+O\left(\alpha^{3}\right)}$ and clearly shows that $\Delta$ decreases with increasing $\alpha$. Moreover we find perfect agreement between the exact value of $\Delta$ and our numerical results: $\Delta \approx 3.51$ for $\alpha=0.25$ and $\Delta \approx 2.62$ for $\alpha=0.4$, respectively.

In Fig 4 we give $\Delta$ and the value of $L^{2} / 4$ as function of $m^{2}$ for the $n=0$ GBAdS black holes for $\alpha=0.25$ and $\alpha=0.4$, respectively. For $\alpha \neq 0$, we find that the maximal value of $m^{2}=m_{\max }^{2}$ is again connected to the saturation of the $\mathrm{AdS}_{2} \mathrm{BF}$ bound $m^{2}=-1 /\left(L^{2}-4 \alpha\right)$ in the extremal limit with $r_{+}=r_{+}^{(\mathrm{ex})}=L / \sqrt{2}$. With our choice $r_{+}=1$ which corresponds to $L=\sqrt{2}$ for the extremal solution we find $m_{\max }^{2}=-1$ for $\alpha=0.25$ and $m_{\max }^{2}=-2.5$ for $\alpha=0.4$. This is in good agreement with the results shown in Fig⿴囗十 and can also be seen from the curves for $L^{2} /(4 \alpha)$. In the extremal limit we have $L=\sqrt{2}$ with our choice $r_{+}=1$. Hence, $L^{2} /(4 \alpha)=2$ for $\alpha=0.25$ and $L^{2} /(4 \alpha)=1.25$ for $\alpha=0.4$ for the largest possible value of $m^{2}$. This is in perfect agreement with our numerical results. We also find that there is a minimal value of $m^{2}=m_{\min }^{2}$ up to where scalar condensation appears. This can be understood when looking at the curves $L^{2} /(4 \alpha)$. These end at $L^{2} /(4 \alpha)=1$, which corresponds to the Chern-Simons limit. For $L^{2} /(4 \alpha)>1$ there exists no black hole interpretation of the solutions, hence it is natural that these curves end here. For the minimal value of $m^{2}=m_{\min }^{2}$ we find that $m_{\min }^{2} \approx-7.2$ for $\alpha=0.25$ and $m_{\min }^{2} \approx-4.6$ for $\alpha=0.4$. We hence observe that the interval of $m^{2}$ on which the scalar condensation instability appears decreases considerably when including the GB term.

\subsection{Non-linear case: $\gamma \neq 0$}

For $\gamma \neq 0$, we have to solve the full set of equations (4)-(6) subject to the appropriate boundary conditions. The scalar field backreacts onto the space-time in this case. As for $\gamma=0$ we also find that solutions with $n$ nodes of the scalar field function exist. However, we will only present our results for the $n=0$ solutions in this section.

As mentioned earlier we can absorb $\gamma$ into the normalisation of $\psi(r)$ by rescaling $\psi(r) \rightarrow \psi(r) / \sqrt{\gamma}$. We can hence set $\gamma=1$ without loss of generality and label the solution by the value of $\psi_{0}$. Solving the equations for fixed values of $\Delta$ and different values of $\psi_{0}$ we find a family of black hole solutions with a definite relation between $\psi_{0}$ and the AdS radius $L$. Again, we have first reinvestigated the $\alpha=0$ case and our results are shown in Fig 5 , where we give the dimensionless entropy $S / L^{3}$, the dimensionless energy $E / L^{2}$ and the value of $\psi_{0} / L^{\Delta}$ 


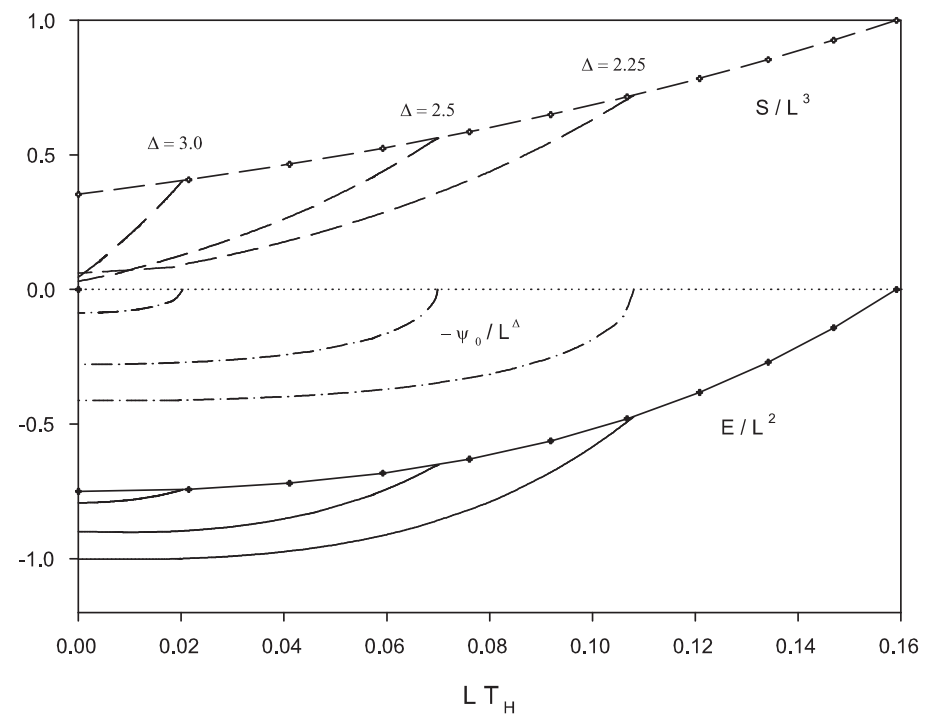

Figure 5: The dimensionless entropy $S / L^{3}$ (dashed), the dimensionless energy $E / L^{2}$ (solid) and the value of $\psi_{0} / L^{\Delta}$ (dotted-dashed) are given as function of the dimensionless temperature $L T_{\mathrm{H}}$ for different values of $\Delta$ and $\alpha=0$. The dashed and solid lines with additional small black dots correspond to the entropy and energy of the exact solution (17) which has $\psi(r) \equiv 0$. Here $\gamma=1$.

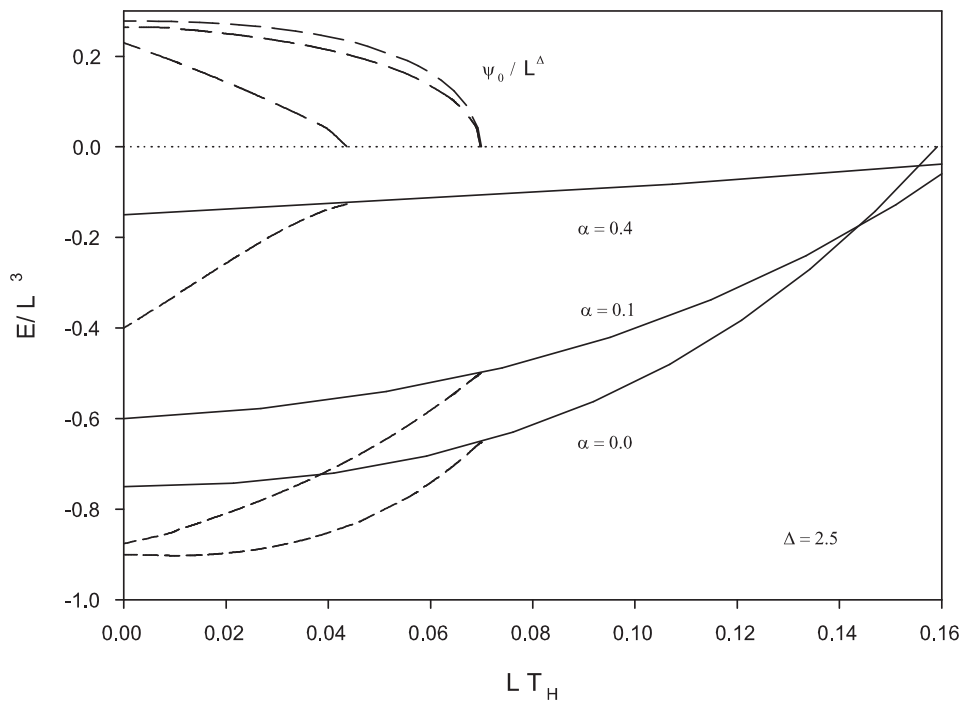

Figure 6: The dimensionless energy $E / L^{2}$ of the exact solution (17) (solid) as well as of the black hole solutions with scalar hair (short-dashed) is given as function of the dimensionless temperature $L T_{\mathrm{H}}$ for $\Delta=2.5$ and different values of $\alpha$. Also shown is the value of $\psi_{0} / L^{\Delta}$ (long-dashed) which becomes non-equal to zero at the value of $L T_{\mathrm{H}}$ at which the short-dashed curves branch of from the solid curves. This corresponds to the onset of the scalar condensation instability. Here $\gamma=1$. 


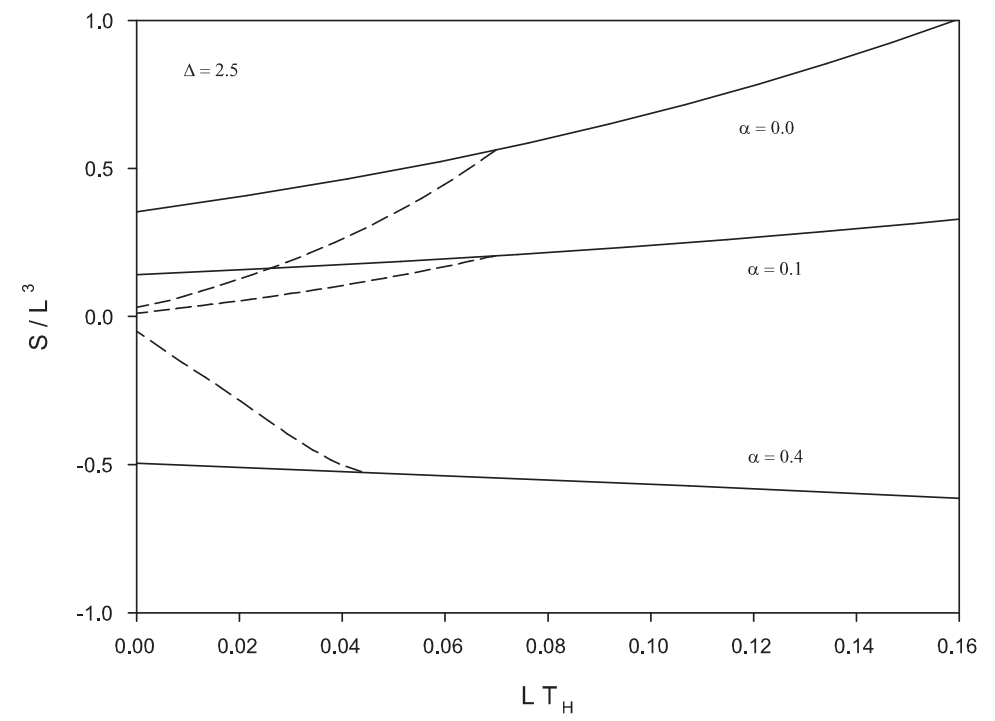

Figure 7: The dimensionless entropy $S / L^{3}$ of the exact solution (17) (solid) as well as of the black hole solutions with scalar hair (short-dashed) are given as function of the dimensionless temperature $L T_{\mathrm{H}}$ for $\Delta=2.5$ and different values of $\alpha$. The short-dashed curves branch of from the solid curves at the onset of the scalar condensation instability. Here $\gamma=1$.

as function of the dimensionless Hawking temperature $L T_{\mathrm{H}}$ for different values of $\Delta$. The case $\Delta=2$ has been given in [25. Here we present the black hole quantities for $\Delta=2.25, \Delta=2.5$ and $\Delta=3$, respectively. We find that at the onset of the scalar condensation instability which corresponds to the value of $L T_{\mathrm{H}}=\left(L T_{\mathrm{H}}\right)_{\mathrm{cr}}$ at which $\psi_{0}$ becomes non-vanishing the curves for the entropy $S / L^{3}$ and entropy $E / L^{2}$ branch of from the curves of the exact solution given by (17), $a(r) \equiv 1$ and $\psi(r) \equiv 0$. We find that the higher the value of $\Delta$ the lower $\left(L T_{\mathrm{H}}\right)_{\mathrm{cr}}:\left(L T_{\mathrm{H}}\right)_{\mathrm{cr}} \approx 0.11$ for $\Delta=2.25,\left(L T_{\mathrm{H}}\right)_{\mathrm{cr}} \approx 0.07$ for $\Delta=2.5$ and $\left(L T_{\mathrm{H}}\right)_{\mathrm{cr}} \approx 0.02$ for $\Delta=3$. To state it differently: the stronger we want the scalar field to fall off on the AdS boundary, the closer we have to choose the solution to be to the extremal solution with $T_{\mathrm{H}}=0$. All solutions with scalar hair have entropy $S / L^{3}$ and energy $E / L^{2}$ smaller than the solutions without hair which suggests that the solutions without scalar hair are thermodynamically favoured. In the limit $T_{\mathrm{H}}=0$ we find in agreement with 25 , that $S / L^{3}=0$. Hence, the hairy black hole should not be interpreted as extreme black hole solutions in this limit 25 .

In Fig 6 and Fig 7 we give the dimensionless entropy $S / L^{3}$, the dimensionless energy $E / L^{2}$ and the value of $\psi_{0} / L^{\Delta}$ as function of the dimensionless Hawking temperature $L T_{\mathrm{H}}$ for different values of $\alpha$ and $\Delta=2.5$. Again, the curves for the energy $E / L^{2}$ and the entropy $S / L^{3}$ branch of from those for the exact solution (17) at the onset of the scalar condensation instability at which $\psi_{0}$ becomes non-vanishing. Note that we use the "standard" definition for $S$ making the entropy negative for certain GBAdS black holes. We could substract the entropy of the $M=0$ solution from this as was suggested in [11, but this would not change the qualitative results and moreover is still an open question. Our numerical results suggest that the larger $\alpha$ the smaller $\left(L T_{\mathrm{H}}\right)_{\mathrm{cr}}$ at which scalar condensation appears: $\left(L T_{\mathrm{H}}\right)_{\mathrm{cr}} \approx 0.07$ for $\alpha=0,\left(L T_{\mathrm{H}}\right)_{\mathrm{cr}} \approx 0.065$ for $\alpha=0.25$ and $\left(L T_{\mathrm{H}}\right)_{\mathrm{cr}} \approx 0.029$ for $\alpha=0.4$. Stating it differently: the larger $\alpha$ the harder it gets to make an uncharged scalar field condense on a black hole. This has already been observed in the case of charged scalar fields in the context of holographic superconductors [27, 28]. 


\section{Conclusions}

In this paper we have studied the condensation of an uncharged scalar field on uncharged hyperbolic SAdS and GBAdS black holes in $(4+1)$ dimensions. These black holes possess an extremal limit such that the near horizon geometry contains an $\mathrm{AdS}_{2}$ factor. We confirm that the onset of scalar condensation appears for scalar field mass equal to the $\mathrm{AdS}_{2} \mathrm{BF}$ bound at the extremal solution. Near extremal black holes can also form a scalar condensate, however, the fall-off of the scalar field on the AdS boundary is the slower the further we move away from extremality. We find that a family of hairy black hole solutions exists which is labelled by the number of nodes of the scalar field function. We observe that the higher the number of nodes the closer we have to be to extremality to find scalar field condensation. Moreover, scalar fields with nodes can only condense on reasonably small black holes. We also observe that the inclusion of the GB term makes scalar field condensation harder and restricts the range of masses of the scalar field for which hairy black holes exist considerably.

In 25] static, hyperbolic SAdS black holes were studied as toy model for rotating black holes. It was shown in 34] that any extremal black hole admits a near-horizon limit. Hence, we would expect that for rotating, near-extremal GB black holes in asymptotically flat space similar instabilities against scalar condensation would appear and this is currently under investigation. Moreover, it would be interesting to see whether there exist also hairy black holes with charged scalar fields that contain nodes. Within the context of holographic superconductors these different black holes could be the gravity duals of different superconducting phases at low enough temperature.

Acknowledgments YB would like to thank the Belgian FNRS for financial support.

\section{References}

[1] B. Zwiebach, Phys. Lett. B156 (1985) 315; R. I. Nepomechie, Phys. Rev. D32 (1985) 3201.

[2] D. G. Boulware, S. Deser, Phys. Rev. Lett. 55 (1985) 2656.

[3] J. T. Wheeler, Nucl. Phys. B268 (1986) 737.

[4] D. L. Wiltshire, Phys. Rev. D38 (1988) 2445.

[5] R. -G. Cai, Phys. Rev. D65 (2002) 084014, arXiv:hep-th/0109133.

[6] M. Cvetic, S. 'i. Nojiri, S. D. Odintsov, Nucl. Phys. B628 (2002) 295, arXiv:hep-th/0112045.

[7] Y. M. Cho, I. P. Neupane, Phys. Rev. D66 (2002) 024044, arXiv:hep-th/0202140.

[8] I. P. Neupane, Phys. Rev. D67 (2003) 061501, arXiv:hep-th/0212092.

[9] R. -G. Cai, Q. Guo, Phys. Rev. D69 (2004) 104025, arXiv:hep-th/0311020.

[10] I. P. Neupane, Phys. Rev. D69 (2004) 084011, arXiv:hep-th/0302132.

[11] T. Clunan, S. F. Ross, D. J. Smith, Class. Quant. Grav. 21 (2004) 3447, arXiv:gr-qc/0402044.

[12] see e.g. O. Aharony, S. S. Gubser, J. M. Maldacena, H. Ooguri and Y. Oz, Phys. Rept. 323 (2000) 183, arXiv:hep-th/9905111; E. D'Hoker and D. Z. Freedman, Supersymmetric gauge theories and the AdS/CFT correspondence, arXiv:hep-th/0201253]; M. Benna and I. Klebanov, Gauge-string duality and some applications [arXiv: 0803.1315 [hep-th]].

[13] J. Maldacena, Adv. Theo. Math. Phys. 2 (1998) 231; Int. J. Theor. Phys. 38 (1999) 1113, arXiv:hep-th/9711200.

[14] S. S. Gubser, Phys. Rev. D78 (2008) 065034, arXiv:0801.2977 [hep-th]]. 
[15] S. A. Hartnoll, C. P. Herzog and G. T. Horowitz, Phys. Rev. Lett. 101 (2008) 031601, arXiv:0803.3295 [hep-th]]; JHEP 0812 (2008) 015, arXiv:0810.1563 [hep-th]].

[16] G. T. Horowitz and M. M. Roberts, Phys. Rev. D78 (2008) 126008, arXiv:0810.1077 [hep-th]].

[17] for reviews see C. P. Herzog, J. Phys. A42 (2009) 343001; S. A. Hartnoll, Class. Quant. Grav. 26 (2009) 224002, arXiv:0903.3246 [hep-th]]; G. Horowitz, Introduction to holographic superconductors, arXiv:1002.1722 [hep-th]]; S. A. Hartnoll, Horizons, holography and condensed matter, arXiv:1106.4324 [hep-th]].

[18] P. Breitenlohner and D. Z. Freedman, Annals Phys. 144 (1982) 249.

[19] I. Robinson, Bull. Acad. Pol. Sci. Ser. Sci. Math. Astron. Phys. 7 (1959) 351.

[20] B. Bertotti, Phys. Rev. 116 (1959) 1331.

[21] J. M. Bardeen, G. T. Horowitz, Phys. Rev. D60 (1999) 104030, arXiv:hep-th/9905099.

[22] A. Sen, JHEP 0509 (2005) 038, arXiv:hep-th/0506177.

[23] A. Sen, JHEP 0811 (2008) 075, arXiv:0805.0095 [hep-th]].

[24] O. J. C. Dias, P. J. Silva, Phys. Rev. D77 (2008) 084011, [arXiv:0704.1405 [hep-th]].

[25] O. J. C. Dias, R. Monteiro, H. S. Reall, J. E. Santos, JHEP 1011 (2010) 036, arXiv:1007.3745 [hep-th]].

[26] N. D. Mermin, H. Wagner, Phys. Rev. Lett. 17 (1966) 1133; S. Coleman, Commun. Math. Phys. 31 (1973) 259.

[27] R. Gregory, S. Kanno, J. Soda, JHEP 0910 (2009) 010, arXiv:0907.3203 [hep-th]].

[28] Y. Brihaye, B. Hartmann, Phys. Rev. D81 (2010) 126008, arXiv:1003.5130 [hep-th]].

[29] L. Barclay, R. Gregory, S. Kanno, P. Sutcliffe, JHEP 1012 (2010) 029, arXiv:1009.1991 [hep-th]].

[30] D. Astefanesei, N. Banerjee, S. Dutta, JHEP 0811 (2008) 070, arXiv:0806.1334 [hep-th]].

[31] J. D. Brown, J. W. York, Jr., Phys. Rev. D47 (1993) 1407, arXiv:gr-qc/9209012.

[32] Y. Brihaye, E. Radu, JHEP 0809 (2008) 006, arXiv:0806.1396 [gr-qc]].

[33] U. Ascher, J. Christiansen and R. D. Russell, Math. Comput. 33 (1979), 659; ACM Trans. Math. Softw. 7 (1981), 209.

[34] H. S. Reall, Phys. Rev. D68 (2003) 024024; [Erratum-ibid. D70 (2004) 089902], arXiv:hep-th/0211290.

\section{A Appendix: Extremal solutions}

Let us assume that an extremal limit for black hole solutions in $\mathrm{AdS}_{5}$ exists such that the near horizon geometry is given by $\mathrm{AdS}_{2} \times M_{3}$, where $M_{3}$ can be a manifold of positive curvature $(k=1)$, negative curvature $(k=-1)$ or zero curvature $(k=0)$. Let the volume of $M_{3}$ be denoted by $V_{3}$. Note that only hyperbolic GBAdS and SAdS black holes $(k=-1)$ possess an extremal limit. For the cases $k=1$ and $k=0$, however, one could add an electric charge to obtain an extremal limit. The entropy function formalism [22, 23, 24] was used for charged GBAdS black holes with spherical horizon $(k=1)$ in 30 . Here, we will extend these results to the cases $k=0$ and $k=-1$ keeping in mind that we are interested in the uncharged limit with $k=-1$ in this paper. Following [30] we write the metric of the $\mathrm{AdS}_{2} \times M_{3}$ space-time as

$$
d s^{2}=v_{1}\left(-\rho^{2} d \tau^{2}+\frac{1}{\rho^{2}} d \rho^{2}\right)+v_{2} d \Sigma_{k, 3}^{2}
$$


where

$$
d \Sigma_{k, 3}^{2}= \begin{cases}d \Omega_{3}^{2}=d \psi^{2}+\sin ^{2} \psi\left(d \theta^{2}+\sin ^{2} \theta d \varphi^{2}\right) & \text { for } k=1 \\ d x^{2}+d y^{2}+d z^{2} & \text { for } k=0 \\ d \Xi_{3}^{2}=d \psi^{2}+\sinh ^{2} \psi\left(d \theta^{2}+\sin ^{2} \theta d \varphi^{2}\right) & \text { for } k=-1\end{cases}
$$

The entropy function is $F\left(v_{1}, v_{2}, e, Q\right) \propto Q e-f\left(v_{1}, v_{2}, e\right)$, where $f\left(v_{1}, v_{2}, e\right)$ corresponds to the integral of the action density over the coordinates of $M_{3}$ :

$$
f\left(v_{1}, v_{2}, e\right)=\int_{M_{3}} \sqrt{-g} \tilde{\mathcal{L}} d^{3} x .
$$

$\tilde{\mathcal{L}}$ is the Lagrangian density of Einstein-Gauss-Bonnet-Maxwell theory in AdS

$$
\tilde{\mathcal{L}}=\left(R-2 \Lambda+\frac{\alpha}{2}\left(R^{M N K L} R_{M N K L}-4 R^{M N} R_{M N}+R^{2}\right)-F_{M N} F^{M N}\right), M, N, K, L=0,1,2,3,4 .
$$

$F_{M N}$ is the field strength tensor of a $\mathrm{U}(1)$ gauge field, which we will choose as $F_{\tau \rho}=-F_{\rho \tau}=e[30$. Inserting the metric and the field strength tensor we find

$$
f\left(v_{1}, v_{2}, e\right)=V_{3}\left(6 k v_{1} \sqrt{v_{2}}-2 v_{2}^{3 / 2}+v_{1} v_{2}^{3 / 2} \frac{12}{L^{2}}-12 k \alpha \sqrt{v_{2}}+2 e^{2} \frac{v_{2}^{3 / 2}}{v_{1}}\right),
$$

where $V_{3}$ corresponds to the integral over the coordinates of $M_{3}$, which for $k=1$ is $V_{3}=2 \pi^{2}$, while we set $V_{3}=1$ for $k=0$ and $k=-1$ such that all quantities that contain this factor are given per unit volume.

The attractor equations are

$$
\begin{aligned}
& \frac{\partial F}{\partial v_{1}}=0 \quad \rightarrow \quad 6 k v_{1}^{2}+\frac{12}{L^{2}} v_{1}^{2} v_{2}-2 e^{2} v_{2}=0 \\
& \frac{\partial F}{\partial v_{2}}=0 \quad \rightarrow \quad k v_{1}^{2}-v_{1} v_{2}+\frac{6}{L^{2}} v_{1}^{2} v_{2}-2 k \alpha v_{1}+e^{2} v_{2}=0 \\
& \frac{\partial F}{\partial e}=0 \quad \rightarrow \quad Q=4 V_{3} e \frac{v_{2}^{3 / 2}}{v_{1}}
\end{aligned}
$$

which reduces to the equations given in [30] for $k=1$. With the identifications $v_{2}=r_{+}^{2}, v_{1}=R^{2}$, where $R$ is the radius of the $\mathrm{AdS}_{2}$ we then find

$$
\frac{Q^{2}}{16 V_{3}^{2}}=3 r_{+}^{4}\left(k+\frac{2 r_{+}^{2}}{L^{2}}\right)
$$

and

$$
R^{2}=\frac{r_{+}^{2}+2 k \alpha}{4 k+12 r_{+}^{2} / L^{2}}
$$

Note that $Q$ is strictly positive for $k=0, k=1$, which relates to the statement above that extremal black holes with flat or spherical horizons exist only if charge is included. On the other hand, for hyperbolic horizons, i.e. $k=-1$ we can set $Q=0$ and find that the value of the horizon is at $r_{+}=L / \sqrt{2}$. We hence find the following values for an extremal GBAdS black hole with hyperbolic horizon and vanishing charge that possesses a near horizon geometry of $\mathrm{AdS}_{2} \times M_{3} \equiv \mathrm{AdS}_{2} \times H^{3}$ :

$$
Q=0, r_{+}=L / \sqrt{2}, \quad R^{2}=\frac{L^{2}}{4}-\alpha .
$$

For $\alpha=0$ this reduces to the well-known SAdS limit with $R=L / 2$. 